\title{
Transient Exposure to 450 nm Light Alters Phagocytosis by Retinal Pigment Epithelium Without Compromising Viability
}

\author{
Jonathan Blaize $^{1 *}$ and Joseph Fabozzi ${ }^{1}$ \\ 1. Department of Biological Sciences, Wagner College, Staten Island, New York, USA. \\ * Corresponding author: Jonathan.Blaize@wagner.edu
}

Phagocytosis of outer segments (OS) and reisomerization of chromophores by the retinal pigment epithelium (RPE) are critical for maintaining visual health [1,6,7,12]. Diseases of the retina, including age-related macular degeneration (AMD), manifest when the aforementioned processes are perturbed or RPE viability is compromised $[8,10,11,14]$. Numerous factors, hereditary or otherwise, have been implicated as contributors to RPE failure though none are as paradoxical as light exposure since it is required for normal vision. While melanosomes within RPE offer protection from photo-oxidative stressors, chronic irradiation contributes to toxic biretinoid production and accumulation $[1,4,6,12,13]$. The retina is particularly vulnerable to short electromagnetic wavelengths $(<500 \mathrm{~nm})$ and recent studies suggest that increased mobile electronic device usage contributes to disease phenotypes [3,12,13]. Several laboratories have demonstrated photo-toxicity of blue light on RPE, though little is known about the consequences of short-term exposure on phagocytosis $[1,3,12,13]$.

The popularity of mobile communication has driven immense progress in display panel technology, however, comprehensive studies of the most popular devices show that manufacturers do little to evaluate or reduce harmful spectral emissions [3]. While the impacts of increased exposure to modern displays on retinal health remain largely unknown, the photochemical damage endured by the retina over time is well characterized $[6,12,13]$.

Lipofuscin production and accumulation is a seemingly unavoidable consequence of phototransduction and can be readily observed in aged RPE [15]. The reason for this accumulation has not been completely elucidated, however, one form of AMD, Stargardt's disease, has been linked to dysfunction of the ABCA4 transport protein responsible for removing the fornamed byproduct [8]. The main constituent of lipofuscin, $N$-retinyl- $N$-retinylidene ethanolamine (A2E), is lipophilic cytotoxin that has been shown to reduce cellular viability, slow phagolysosomal digestion of OS and hinder phagocytosis under certain physiological conditions where oxidative phosphorylation mechanisms are stressed $[1,15]$.

OS clearance by RPE requires activation of signal transduction cascades specific to photoreceptor renewal [6-11,14]. Recognition and internalization of distal photoreceptor targets is facilitated by direct and indirect docking of terminal OS phosphatidylserine patches to receptor tyrosine kinases' (RTK) and apical integrins expressed by RPE, respectively[8,14]. These events trigger RTK activation which invokes multiple signaling pathways, culminating in an increase of cytoplasmic calcium and cytoskeletal reorganization to form the phagocytic cup. Given the propensity of A2E to interfere with the these processes [6], we suspect that brief, sub-lethal exposure to $450 \mathrm{~nm}$ light is sufficient to reduce phagocytic activity and that previously unreported signaling cascades involved in OS clearance are disrupted $[9,12,15]$.

To evaluate changes in RPE phagocytosis induced by transient blue light exposure, ARPE-19 were cultured in DMEM-F12 until $80 \%$ confluent and then exposed to $450 \mathrm{~nm}$ light for 15 minutes. 
Phagocytosis was evaluated using a zymosan engulfment assay (Abcam Phagocytosis Assay, Zymosan substrate: ab211156). Cellular morphology and caspase prevalence (Fisher Scientific, rabbit monoclonal antibody, BD559565), a marker of toxicity, were assessed using scanning electron (Topcon ABT-32) and confocal microscopy (Nikon PCM2000), respectively. A MTT (Abcam MTT: ab2011091) assay was employed to evaluate cellular viability as a function of mitochondrial output. Preliminary results support a hypothesis of phagocytosis perturbation when cells are exposed to blue light, ( 0.027 micromole), and that the duration and intensity of this exposure does not alter morphology, induce apoptosis or change mitochondrial output.

\section{References:}

[1] N Boyer et al., Journal of Biological Chemistry 287 (2012), p. 22276.

[2] A Chen et al., PLOS ONE 7 (2012), p. 1.

[3] AJ Clark et al., OSLI Retina 49 (2018), p. 346.

[4] S Datta et al., Progress in retinal and eye research 60 (2017), p. 201.

[5] DL Dillehay et al., Journal of Leukocyte Biol. 44 (1988), p. 353.

[6] P Geiger et al., Cell Death \& Disease 6 (2015), p. 1.

[7] A Iriyama et al., Journal of Biological Chemistry 283 (2008), p. 11947.

[8] BM Kevany and K Palczewski, Physiology 25 (2010), p. 8.

[9] TL Lenis et al., PNAS 115 (2018), p. E11120.

[10] Y Mao and SC Finnemann, Journal of Small GTPases 6 (2015), p. 89.

[11] F Mazzoni et al., Experimental Eye Research 126 (2014), p. 51.

[12] K Ratnayake et al., Nature. Sci. Reports 8 (2018), p. 1.

[13] C Roehlecke et al., Molecular Vision 15 (2009), p. 1929.

[14] O Strauss, Physiological Review 85 (2005), p. 845.

[15] C Vives-Bauza et al., Journal of Biological Chemistry 283 (2008), p. 24770.

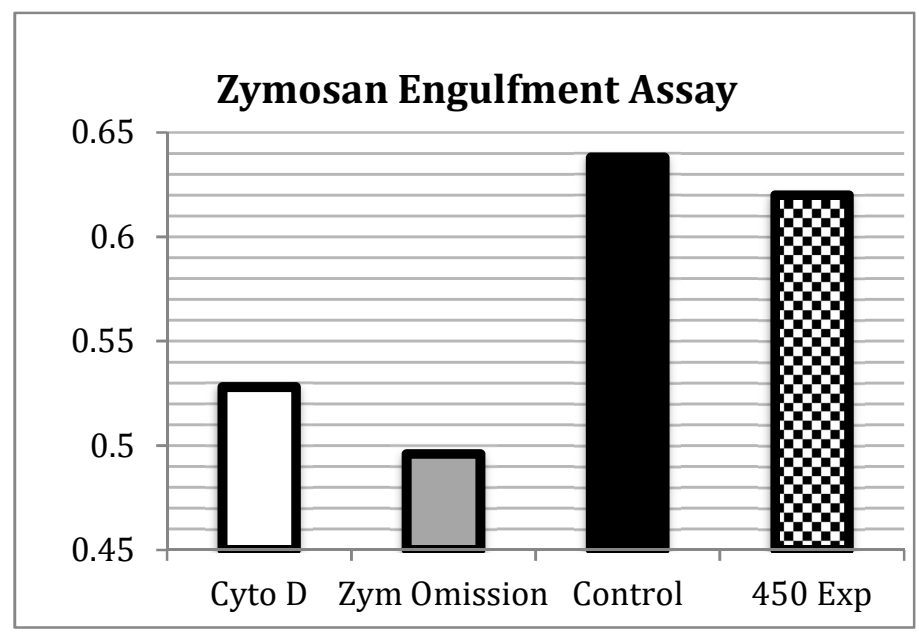

Figure 1. Spectrophotometry was used to quantify zymosan engulfment by RPE. A reduction in phagocytosis, interpreted as absorption (405 nm), is observed when ARPE-19 were exposed to $450 \mathrm{~nm}$ light prior to zymosan challenge. Cytochalasin D and omission of zymosan particles were used as negative controls. 